\title{
V. Chondrostibian, ein neues Mineral von der Manganerzgrube Sjögrufvan, Kirchspiel Grythyttan, Gouv. Örebro, Schweden.
}

Von

L. J. Igelström in Sunnemo.

Im Januar d. J. gelang es mir, noch ein neues Antimonmineral in der bereits durch das Vorkommen der Mineralien Hämatostibiit, Ferrostibian, Stibiatil, Basiliit und Melanostibian bekannten Sjögrube zu entdecken. Ich

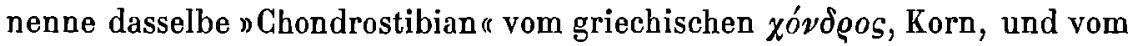
lateinischen Stibium, Antimon, weil es in Körnern vorkommt. Die kleinen Körner finden sich in Schwerspath eingestreut und scheinen zuweilen Kryställchen von oktaëdrischer Form zu bilden.

Der die Ghondrostibiankörnchen und Kryställchen umgebende Baryt bildet blätterige Partien in einem kryptokrystallinischen Gemenge von Schwerspath, Galcit oder Dolomit und Tephroit. Die deutlich grossblätterigen Partien gehen aber nach und nach in das kryptokrystallinische Gemenge über, in welchem sich ebenfalls Chondrostibiankörnchen finden, welche nur dem Schwerspath anzugehören scheinen. In dem Gemenge sind im Allgemeinen die Chondrostibiankörnchen sehr zerstreut, aber zuweilen erscheinen sie in grösserer Zahl beisammen und bilden so reichere Adern von $2-5 \mathrm{~cm}$ Breite.

Der blätterig abgesonderte Schwerspath enthält etwa $50 \%$ reines Mineral und erscheint durch dasselbe braunroth gefärbt, so dass er einem braunrothen Kalkspath ähnelt. Wenn man eine Barytlamelle unter das Mikroskop legt, so sieht man die kleinen Körnchen des Minerals verstreut in sebr grosser Menge und sehr nahe aneinander in dem wasserklaren, durchsichtigen Schwerspath. Das Mineral selbst ist in kleinen Körnchen mit gelbrother Farbe durchsichtig. In grösseren Körnern erscheint es im reflectirten Lichte dunkel braunroth. Das Pulver des Gemenges mit Schwerspath ist braunroth bis chocoladebraun. An der Luft gegluht wird es schwarz unter Verlust von viel Wasser. Ausserdem enthält der grossblätterige 
Schwerspath nur Spuren von Dolomit zwischen seinen Lamellen und vielleicht Spuren von Tephroit (?). Er gelatinirt daher nicht mil Chlorwasserstoffsäure, während dies der Fall ist mit dem kryptokrystallinischen Gemenge wegen seines grossen Gehaltes an Tephroil. Aus dem einen wie dem anderen lässt sich der Chondrostibian mit letztgenannter Säure ausziehen, wenn auch sehr schwer. Die Lösung ist gelblich und enthält ziemlich viel Antimonsäure neben Mangan und Eisen. Bei der Auflösung entwickelt sich viel Chlor, daher das Mangan in dem Mineral in einer höheren Oxydationsstufe zugegen sein muss.

Da ich nicht hinreichende Quantitäten des blälterigen Schwerspathes hatte und ein Auslesen der Körnchen des Minerals mit der Pincette kaum möglich sein möchte, musste ich die quantitative Analyse mit dem Material der obengenannten reichen Adern ausfubren. Diese Adern wurden - was man scbon aus theoretischen Gründen schliessen konnte - als aus Schwerspath, Dolomit, Tephroit und Chondrostibian bestehend gefunden (siehe die unten stehende quantitative Analyse).

Neben dem Chondrostibian findet sich übrigens in der Sjögrube Melanostibian und noch ein anderes nicht näher untersuchtes Stibiat von schwarzer Farbe, magnetisch (sehr schwach), in kleinen Kryställchen (Oktaëdern), Drusen in den Hohlräumen des Gemenges bildend. Dasselbe ist ebenfalls wasserhaltig.

Der Ghondrostibian ist sehr schwach magnetisch. Er giebt im Kölbchen viel Wasser, schmilzt sehr schwer zu einer schwarzen Kugel, giebt etwas Antimonrauch und einen schwachen Geruch von Arsen. Der Antimonrauch ist jedoch nicht so stark wie beim Melanostibian. Die von mir zur quanlitativen Analyse verwendete Probe schwärzte Silber sehr stark nach der Reduction mit Soda auf Kohle und nach der Befeuchtung der Masse mit Wasser. So verhielt sich auch der bei meiner Analyse als ungelöst abgeschiedene Theil. Diese Schwärzung kommt jedoch nicht von dem Chondrostibian selbst her, sondern von dem immer damit vergesellschafteten Schwerspath.

Die Altersfolge der vier in dem Gemenge vorhandenen Mineralien ist deutlich die folgende: 1. Chondrostibian; 2. Schwerspath; 3. Tephroit; 4. Dolomit. Das noch nicht näher untersuchte, im Chondrostibian in Drusen auftretende schwarze wasserhaltige Stibiat ist noch jünger als der Chondrostibian, ebenso der Melanostibian, der in reinen Adern in dem Gestein erscheint.

$1,01 \mathrm{~g}$ von einer der oben erwähnten Adern gab mir: 


$\left.\begin{array}{lc}\mathrm{gaO}, \mathrm{CO}_{2} & 0,0710 \\ \mathrm{MgO}, \mathrm{CO}_{2} & 0,0160 \\ \text { Unlöslich } & 0,1900\end{array}\right\} 0,2770$

Nachdem der bei dieser Analyse gefundene in Chlorwasserstoffsäure unlösliche Theil für sich analysirt und nachdem alles $\mathrm{Mn}_{3} \mathrm{O}_{4}$ als $\mathrm{Mn}_{2} \mathrm{O}_{3}$ berechnet worden war, ergab sich:

$\left.\begin{array}{lrc} & \multicolumn{2}{c}{\mathrm{g}:} \\ \mathrm{CaO}, \mathrm{CO}_{2} \text { und } \mathrm{MgO}, \mathrm{CO}_{2} \text { (Kalkstein) } & 0,0870 \\ \text { Unlöslicher Theil } & \\ \left.\begin{array}{lrl}\mathrm{BaO}, \mathrm{SO}_{3} \text { (Schwerspath) } & 0,0350 & \\ \mathrm{SiO}_{2} \text { (zum Tephroit gehörig) } & 0,1000 & \\ \text { Unzersetztes Stibiat } & 0,0550 & 0,1900\end{array}\right\} \\ \mathrm{Sb}_{2} \mathrm{O}_{5} & 0,15770 \\ \mathrm{As}_{2} \mathrm{O}_{5} & 0,0104 \\ \mathrm{Mn}_{2} \mathrm{O}_{3} & 0,4243 & \\ \mathrm{Fe}_{2} \mathrm{O}_{3} & 0,7566 \\ \mathrm{H}_{2} \mathrm{O} & 0,0945\end{array}\right\}$

Wenn nun das in vorstehender Analyse gefundene $\mathrm{SiO}_{2}$ als zum Tephroit gehörig angesehen wird und der Tephroit als aus $30 \% \mathrm{SiO}_{2}$ und $70 \% \operatorname{MnO}$ zusammengesetzt betrachtet wird, so bleibt schliesslich als Resultat der Analyse:

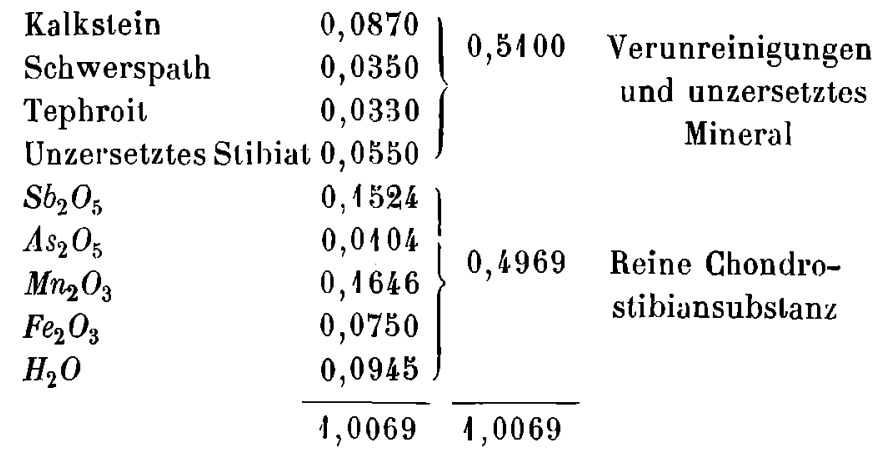


Darnach besteht der Chondrostibian in Procenten aus :

$\begin{array}{lrrr}\mathrm{Sb}_{2} \mathrm{O}_{5} & 30,66 & \text { Sauerstoff } 7,68 \\ \mathrm{As}_{2} \mathrm{O}_{5} & 2,10 & 0,73 & 8,41 \\ \mathrm{Mn}_{2} \mathrm{O}_{3} & 33,13 & 10,10 & \\ \mathrm{Fe}_{2} \mathrm{O}_{3} & 15,10 & 4,53 & 14,63 \\ \mathrm{H}_{2} \mathrm{O} & 19,01 & & 17,65\end{array}$

Hiernach könnte man die chemische Formel :

$$
3 \mathrm{R}_{2} \mathrm{O}_{3} . \mathrm{Sb}_{2} \mathrm{O}_{5}+10 \mathrm{H}_{2} \mathrm{O} \text { oder } 2\left(\mathrm{R}_{2} \mathrm{O}_{3} . \mathrm{Sb}_{2} \mathrm{O}_{5}\right)+4 \mathrm{R}_{2} \mathrm{O}_{3} .5 \mathrm{H}_{2} \mathrm{O}
$$

für den Chondrostibian aufstellen, worin $\mathrm{R}_{2} \mathrm{O}_{3}=\mathrm{Mn}_{2} \mathrm{O}_{3}$ und $\mathrm{Fe}_{2} \mathrm{O}_{3}$ bedeutet und neben $\mathrm{Sb}_{2} \mathrm{O}_{5}$ eine kleine Menge $\mathrm{As}_{2} \mathrm{O}_{5}$ enthalten ist. Aber es muss hier bemerkt werden, dass ein Theil des Eisens als $\mathrm{FeO}$ in dem Mineral vorhanden ist, was durch die magnetische Beschaffenheit des Minerals deutlich angezeigt wird und dass ferner bei meiner Analyse wahrscheinlich das Wasser etwas zu hoch gefunden wurde. Schliesslich mag noch auf die grosse Schwierigkeit, bei gleichzeitiger Gegenwart der Oxydationsstufen des Antimons und Mangans eine vollständige Trennung dieser Stoffe zu erzielen, hingewiesen werden. Indessen ist das Mineral unzweifelbaft ein neues und scheint dem Basiliit am nächsten zu stehen.

Sehr bemerkenswerth ist es, dass in dem Manganerze von Pajsberg ein wasserhaltiges Manganoxydularsenit, der Chondroarsenit, in Schwerspath eingestreut vorkommt. Hier liegt sonach eine Analogie mit dem Vorkommen von Sjögrufvan vor. Diese kann übrigens nicht auffallen, denn Pajsberg und Sjögrufvan sind ja auch analoge Erzlagerstätten : auf beiden kommt Hausmannit vor; beide führen Arseniate und Stibiate, Ghlormineralien (Friedelit, Hedyphan) etc. - Uebrigens dürfte hier erwähnt zu werden verdienen, dass solche Erzvorkommnisse in Schweden sebr selten sind, nämlich nur die folgenden: Långban, Nordmarken, Pajsberg, Jacobsberg (inclusive Harstigen) und Sjögrufvan. Der letztgenannte Fundort zeichnet sich von den anderen durch Vorherrschen von Stibiaten aus.

Sunnemo, 25. April 1893. 